\title{
Antibiotic Optimisation In Vascular Surgery - A Quality Improvement Project
}

\author{
Sarah Jane Messeder*, Bryce Renwick and Alasdair Wilson
}

Department of Vascular Surgery, UK

*Corresponding author: Sarah Jane Messeder, Department of Vascular Surgery, Aberdeen Royal Infirmary, Aberdeen, UK

\section{Introduction}

Surgical infections are a serious cause of morbidity and mortality. Infections within vascular surgery pose a serious threat not only to limb but also to life. For example, graft infection can result in widespread systemic infection, sepsis and death. The incidence of prosthetic graft infections has been shown to vary from 1 to $6 \%$ [1]. However, the morbidity associated is strongly related to the site of surgery and operation performed with studies demonstrating a $21 \%$ early operative mortality and $50 \% 5$-year mortality with an infected prosthetic aortic aneurysm repair [2]. Antibiotic prophylaxis is used within surgery to minimise these complications and ultimately improve mortality. The Scottish Intercollegiate Guidelines Network (SIGN) has assessed the requirement for which vascular operations require antibiotic prophylaxis. In their most recent guideline antibiotic, surgical procedures are grouped into surgical specialities to determine whether antibiotic prophylaxis is recommended, to be considered or not required [3].

Common risk factors for surgical site infections such as poor nutritional status, diabetes mellitus, smoking and extremes of age are also discussed; all common to those suffering with peripheral vascular disease. Analysis of the Society for Vascular Surgery Vascular Quality Initiative Registry from 2003 to 2012 demonstrated an overall in-hospital surgical site infection rate after lower extremity bypass of $4.8 \%$ for 7908 procedures [4]. However, the incidence of surgical site infections been noted to be as high as 32\% [5]. NHS Grampian's 'Antibiotic Prophylaxis in Vascular Surgery' guideline was created based upon the SIGN guideline in conjunction with the Head of Service and the Chair of Antimicrobial Management Team. They determined which antibiotics should be administrated according to the surgery performed and local antimicrobial guidelines. This guideline, found on the NHS Grampian intranet, should be adhered to in all vascular operations. Our aim was to audit the antibiotics used and the timing of administration for all elective vascular surgeries in Aberdeen Royal
Infirmary (ARI) and compare them to the NHS Grampian 'Antibiotic Prophylaxis in Vascular Surgery'.

\section{Materials and Methods}

\section{Ethical Considerations}

A retrospective service data review was carried out in ARI. No ethical approval was required as information was collected for audit purposes only. The audit was approved by the NHS Grampian Quality, Governance and Risk Unit and registered onto the Clinical Effectiveness Database: Project ID 3813.

\section{Data Collection and Analysis}

Data were collected retrospectively from the elective vascular theatre in ARI. Patients were identified using the elective vascular theatre logbook. Only elective patients were included in the audit. The electronic system and paper case records were analysed to obtain the operation note, anaesthetic records, allergies and drug prescription chart. Data collected included: gender, age, antibiotics prescribed prior and during theatre, administration time of antibiotics, allergies and operation and start and finish time of each operation. The first audit cycle ran from February-March 2017 inclusive. The standard assessing what antibiotic prophylaxis is required was compared against NHS Grampian's 'Antibiotic Prophylaxis in Vascular Surgery', which is based upon SIGN 104 guideline [Appendix]. The information audited included antibiotic choice and timing of its administration. The guideline was sent to all vascular consultants, registrars and core trainees as well as the vascular anaesthetists on three separate occasions and placed within the vascular ward doctors' room and theatre. The audit cycle was then repeated from May-June 2017 inclusive. The standard assessing what antibiotic prophylaxis is required was compared against NHS Grampian's 'Antibiotic Prophylaxis in Vascular Surgery', which is based upon SIGN 104 guideline [Appendix 1]. Data were then recorded into a spreadsheet using Microsoft Excel 2013 and analysed. 


\section{Results}

\section{Demographics}

The first audit cycle included a total of 60 elective operations and the second audit cycle included 64 . The patient demographics of both cycles are demonstrated. The operation category was divided up according to the NHS Grampian policy; abdominal and lower limb arterial reconstruction, carotid endarterectomy, lower limb amputation, upper limb renal access and those that did not fit into a specific category.

\section{First Audit Cycle Operations}

In total, $5 \%$ of operations did not correspond to a category within the NHS Grampian guidelines $(n=3)$. This included a biopsy of a foot ulcer and two tie off brachiocephalic fistula operations.

\section{Antibiotics Compared to Guideline}

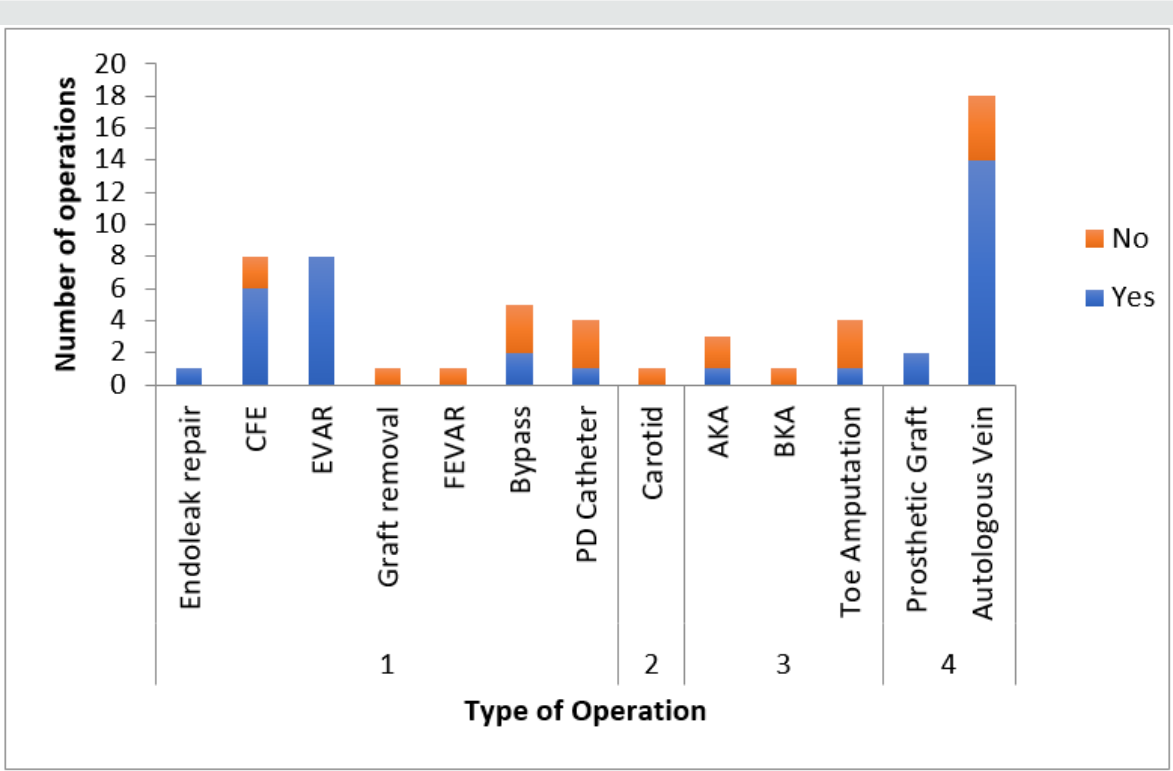

$1=$ Vascular surgery (Abdominal and lower limb arterial reconstruction)

2 = Carotid endarterectomy

3 = Lower Limb amputation

$4=$ Upper limb renal access

$\mathrm{CFE}=$ Common Femoral Endarterectomy, EVAR = Endovascular Aneurysm Repair, FEVAR = Fenestrated Endovascular Aneurysm Repair, PD = Peritoneal Dialysis, AKA = Above Knee Amputation, BKA = Below Knee Amputation

Figure 1: First audit cycle: antibiotics prescribed according to guideline for individual operations.

Of a total of 60 operations, $5 \%$ did not correlate to a category within the guideline $(n=3)$. Of the 57 that did, $63 \%$ received antibiotics according to NHS Grampian guidelines ( $n=36), 21 \%$ were prescribed antibiotics that were different $(n=12)$ and $16 \%$ did not receive antibiotics $(n=9)$. Figure 1 demonstrates a breakdown of individual operation categories according to whether or not the antibiotics prescribed adhered to guideline (Figure 1).

\section{Different Antibiotics}

There were 12 cases that had antibiotics prescribed that were different from the guideline. Of these, $58 \%$ were not compliant due to the avoidance in prescribing gentamicin ( $n=7), 25 \%$ were prescribed benzypenicillin and gentamicin as per guideline but without the addition of flucloxacillin $(\mathrm{n}=3)$ and $8 \%$ missed out appropriate Gram-negative cover $(n=1)$.

\section{Timing of Antibiotics}

Of those operations that received antibiotics, $79 \%$ were administrated 'At induction, $\leq 60$ minutes before incision' as per guideline ( $\mathrm{n}=37), 19 \%$ of antibiotics were given too late $(\mathrm{n}=9)$ and $2 \%$ were given too early $(n=1)$.

\section{Second Audit Cycle}

\section{Operations}

A total of $28 \%$ of all operations did not fit a specific category $(n=18)$. This included a biopsy of a foot ulcer, closure of a fasciotomy, vein operations, antecubital fossa wound debridement, cervical rib resections, thigh loop access graft, incision and drainage of a brachiocephalic fistula abscess and an excision of a prosthetic graft.

\section{Antibiotics Compared to Guideline}

Of the 64 operations, $28 \%$ had no antibiotic guideline $(n=18)$. Of the 46 that did, $63 \%$ received antibiotics according to guideline $(n=29), 28 \%$ had different antibiotics prescribed $(n=13)$ and $9 \%$ did not receive antibiotics $(n=4)$. Figure 2 demonstrates individual operations and their correlation of antibiotics prescribed according to guideline (Figure 2). 


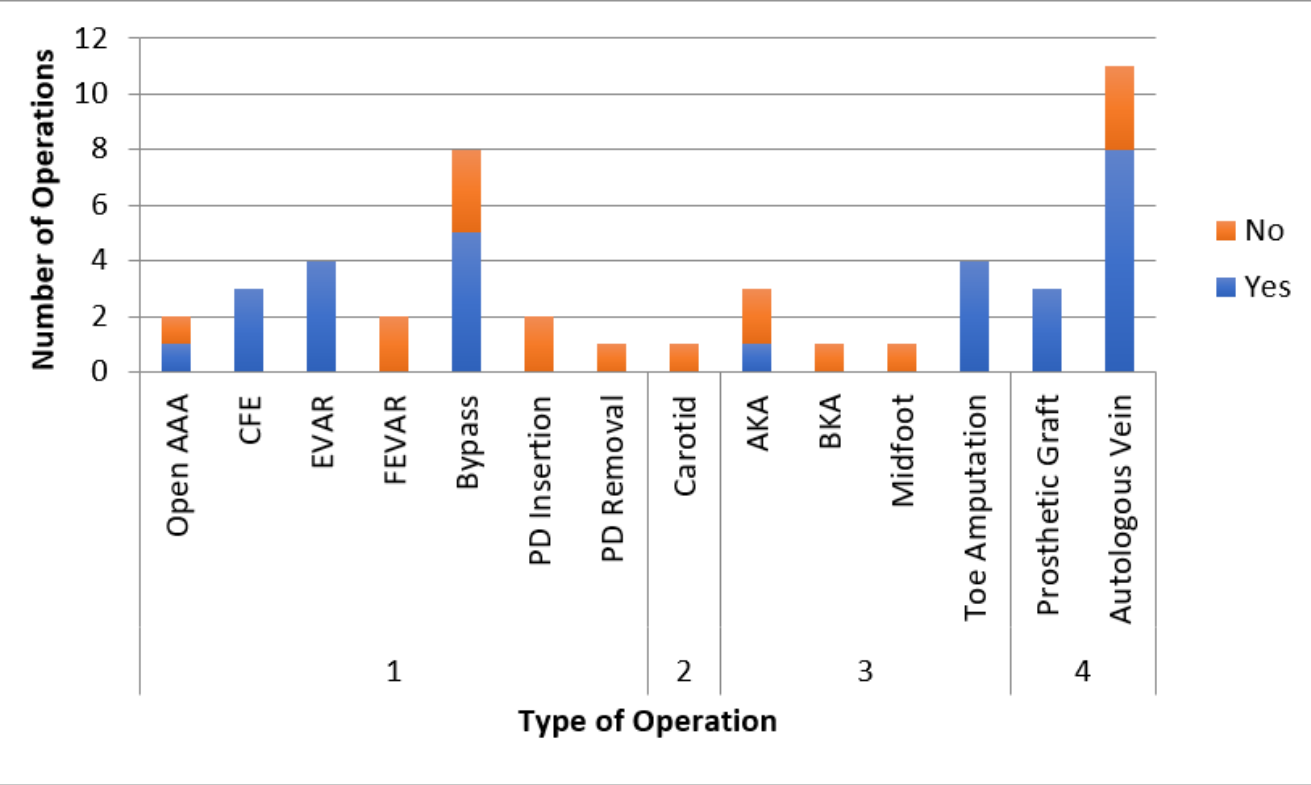

Figure 2: Second audit cycle: antibiotics prescribed according to guideline for individual operation (For abbreviations see Figure 1).

AAA = Aortic Aneurysm Repair

\section{Different Antibiotics}

In total, 12 cases had antibiotics prescribed that were different from guideline. A total of $58 \%$ were not compliant due to the avoidance in prescribing gentamicin $(n=7), 17 \%$ were prescribed benzypenicillin instead of flucloxacillin $(n=2), 8 \%$ missed out the addition of flucloxacillin ( $\mathrm{n}=1)$, $8 \%$ did not have appropriate Grampositive cover $(n=1)$ and $8 \%$ gave gentamicin when it was not needed $(n=1)$.

\section{Timing of Antibiotics}

A total of $88 \%$ of all antibiotics prescribed were administrated according to guideline $(n=30), 9 \%$ of antibiotics were given too late $(n=3)$ and $3 \%$ of antibiotics were given too early $(n=1)$.

\section{Discussion}

The audit reveals important data determining whether local guidelines for antibiotic prophylaxis, during vascular surgery, are being accurately followed. The Getting It Right First Time (GIRFT) is a national programme focused at reducing unwarranted variations and ultimately improving patient care [6].Through the use of local and national collaboration medical professionals are able to analyse results and improve service management. This process is further strengthened by assessing local guidelines and implementing change. Thus, the results of this audit come at a time when post-operative infection rates are at the forefront of the public and governmental interest.

\section{Data Recording}

Accurate documentation is key not only for patient safety but also for correct patient management. NHS England has revealed their 'Five Year Forward View', which outlines the national target that all documentation be electronic by 2020 [7]. This is intended to improve communication between health care providers, allow for 'real-time digital information on a person's health and care', reduce cost and improve patient safety [8]. During the first audit cycle, $15 \%$ of all anaesthetic records and $48 \%$ of all operation notes were not uploaded to the electronic system. These initial results are concerning as accurate documentation enables medical practitioners to record complications making future interactions safer. Additionally, with the current ageing population and demands upon healthcare, clinicians are constantly under pressure to reduce the ever-expanding waiting times. This is often achieved with postoperative appointments carried out by different clinicians and more junior staff. Therefore, it is important that all records be easily accessible. These results were highlighted to clinicians and the second audit cycle saw a vast improvement: only $5 \%$ of anaesthetic records and $17 \%$ of operation notes were not uploaded.

\section{Deviation from Protocol}

Another area highlighted by the audit was the lack of documentation regarding the reasons for not using appropriate guidelines. The GMC states that: 'Clinical records should include relevant clinical findings, the decisions made and actions agreed, and who is making the decisions and agreeing the actions, the information given to patients, any drugs prescribed or other investigation or treatment, who is making the record and when [9]. Thus, decisions regarding differing antibiotic prescriptions require accurate documentation. This allows clinicians to realise that the change from protocol is intended and enables others to understand the rationale behind this. The first audit cycle revealed that $21 \%$ of antibiotics were prescribed against protocol with $16 \%$ of operations receiving no antibiotic cover. Despite clinician awareness of the current NHS Grampian guidelines increasing throughout the audit intervention, the second cycle revealed similar figures with $28 \%$ of antibiotics being prescribing against protocol and $9 \%$ of all operations not receiving antibiotics. 
The insertion and removal of peritoneal dialysis catheters was a common area of wrongful prescribing. Literature strongly links the insertion of peritoneal dialysis without antibiotic prophylaxis with an increased risk of peritonitis [10]. The International Society of Peritoneal Dialysis recommends in their guidelines the use of antibiotic prophylaxis in peritoneal access with strong evidence for vancomycin [11]. No guidelines exist for the removal of a peritoneal dialysis catheter and antibiotic prophylaxis. This recommendation should be considered when updating the current guideline with a separate section added for peritoneal dialysis. Another area that saw a deviation from protocol was when individuals prescribed either benzylpenicillin instead of flucloxacillin or missed out the addition of flucloxacillin when benzylpenicillin was being used. It is unclear why this was the case as accurate documentation was scarce. One could assume that a lack of understanding regarding the differing organisms covered by each antibiotic could play a role. Thus, further investigation into why this occurred and education into why these antibiotics are included in the current guideline should occur.

\section{Gentamicin}

Concerns raised by both the anaesthetic and vascular team included the reluctance to use nephrotoxic antibiotics in procedures associated with high renal injury, such as, those undergoing contrast or requiring clamping of renal vessels. In the current guideline the first- and second-line antibiotic for abdominal and lower limb arterial reconstruction includes gentamicin, a well-known nephrotoxic. 58\% of non-compliance in both the first and second cycle was due to the omission of gentamicin. In these cases, individuals were either prescribed teicoplanin alone or metronidazole instead. Single-dose prophylactic gentamicin has been extensively researched with many articles reporting its safety, as toxicity is associated more with therapeutic level duration rather than peak levels.10 However, a study by Nielsen et al. found an increased incidence of acute kidney injury in patients receiving single-dose prophylactic gentamicin during cardiac surgery, but no greater increase in postoperative dialysis or mortality [12]. When considering the administration of gentamicin clinicians must remember the risk factors for its toxicity: older age, reduced renal function, dehydration and concomitant use of diuretics or iodide contrast media [13]. Importantly, these risk factors are present in a substantial proportion of vascular patients. Interestingly, in the NHS Lanarkshire 'Antibiotic Prophylaxis in Vascular Surgery' guideline, clinicians are asked to consider reducing the dose of gentamicin from $80 \mathrm{mg}$ to $40 \mathrm{mg}$ or omitting altogether in patients who are at risk of developing an acute kidney injury [14]. This poses several questions: is the current $120 \mathrm{mg}$ dose of gentamicin required or is a smaller dose as effective; should we reduce the dose in patients at risk of an acute kidney injury and could other antibiotics with less nephrotoxicity be considered?

\section{Reclassification as Angiogram Procedures}

The risk of surgical site infection (SSI) depends on the type of operation and site. Open procedures carry a greater risk of SSI due to the larger wound created and the exposure of viscera compared to those carried out via angiogram. Skin commensals differ greatly for vascular interventional radiology procedures such as digital subtraction angiograms. Patients within NHS Grampian are most commonly prescribed flucloxacillin and guidelines are currently being developed for these procedures. The argument remains whether patients undergoing Endovascular Aneurysm Repair (EVAR) should be classified according to these interventional guidelines rather than as abdominal and lower limb arterial reconstruction surgery due to the lack of abdominal viscera exposure, thus avoiding the use of nephrotoxics.

\section{Antibiotic Timing}

The timing of antibiotics is important to guarantee efficacy and reduce SSI. The optimal administration of antibiotics is within 60 minutes prior to skin incision with the odds of SSI rising significantly out with this time frame [3]. The correct timing of antibiotic administration improved from $79 \%$ within the first audit cycle to $88 \%$ in the second.

\section{Categorisation}

In the first audit cycle $5 \%$ of operations could not be categorised compared to $28 \%$ of operations within the second. Anterior cervical rib resection for thoracic outlet obstruction is a procedure commonly carried out in ARI, however, this procedure has not been categorised. Similarly, the treatment of varicose veins is commonly carried out and this too fails to be categorised. Although it is not possible to ensure that every procedure fits into an operation category it is important that common operations are grouped so that appropriate antibiotic prophylaxis can be prescribed.

\section{Conclusion}

This audit of antibiotics within intraoperative vascular surgery demonstrates interesting results. A clear improvement has been made in data recording, antibiotic timing and antibiotic coverage. However, the results show no clear difference in antibiotics prescribed according to guideline. This could be explained, in part, by the reluctance of clinicians to prescribe nephrotoxic antibiotics as well as the lack of clear categorization of certain procedures. Thus, an update of the current guideline is required to improve antibiotic prescription and patient care. In an era of Antibiotic Guardianship, it is imperative in order to reduce morbidity and mortality that appropriate antibiotic prescribing is adhered to and practitioners do not become complacent.

\section{References}

1. Wilson WR, Bower TC, Creager MA (2016). Vascular Graft Infections, Mycotic Anuerysms, and Endovascular Infections: A Scientific Statement from the American Heart Association. Circulation 134(20): e412-e460.

2. Oderich GS, Panneton JM, Bower TC (2001) Infected aortic aneurysms: aggressive presentation, complicated early outcome, but durable results. J Vasc Surg 34(5): 900-908.

3. Scottish Intercollegiate Guidelines Network (SIGN). Antibiotic prophylaxis in surgery. Edinburgh: SIGN. 2008. [Accessed 30th January 2019].

4. Kalish JA, Farber A, Homa K (2014) Factors associated with surgical site infection after lower extremity bypass in the Soceity for Vascular Surgery (SVS) Vascular Quality Initiative (VQI). J Vasc Surg 60(5): 12381246.

5. Turtiainen J, Hakala T (2014) Surgical wound infections after peripheral vascular surgery. Scand J Surg 103(4) :226-231. 
6. GIRFT.

7. National Health Service (NHS). Five year forward view. NHS England. 2014

8. National Health Service (NHS). Personalised Health and Care 2020. NHS England. 2014.

9. General Medical Council (GMC). Good medical practice. GMC. 2013.

10. Figueiredo A, Goh BL, Jenkins S (2010) Clinical practice guidelines for peritoneal access. Perit Dial Int. 30(4): 424-429.

11. Li PK, Szeto CC, Piraino B, De Arteaga J, Fan S, et al. (2016) ISPD Peritonitis Recommendations: 2016 Update on Prevention and Treatment. Perit Dial Int 36(5): 481-508.
12. Nielsen DV, Fedosova M, Hjortdal V (2014) Is single-dose prophylactic gentamicin associated with acute kidney injury in patients undergoing cardiac surgery? A matched-pair analysis. J Thorac Cardiovasc Surg 148(4): 1634-1639.

13. Lopez Novoa JM, Quiros Y, Vicente L, Morales AI, Lopez Hernandez FJ (2011) New insights into the mechanism of aminoglycoside nephrotoxicity: an integrative point of view. Kidney Int 79(1): 33-45.

14. National Health Service (NHS). Antibiotic Prophylaxis in Vascular Surgery. NHS Lanarkshire.2017.

\section{(C) (P) \\ This work is licensed under Creative Commons Attribution 4.0 License}

To Submit Your Article Click Here:

Submit Article

DOI: $10.32474 /$ SCSOAJ.2019.02.000144

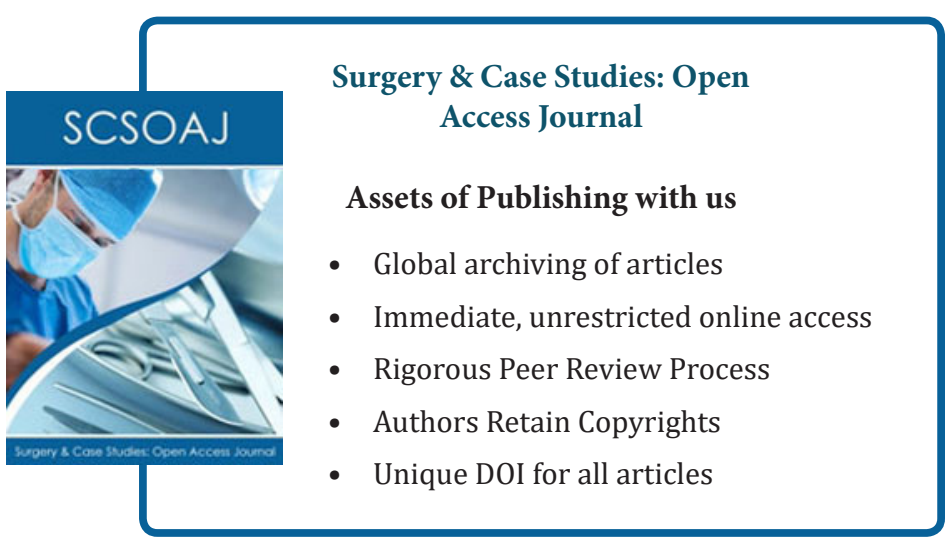

